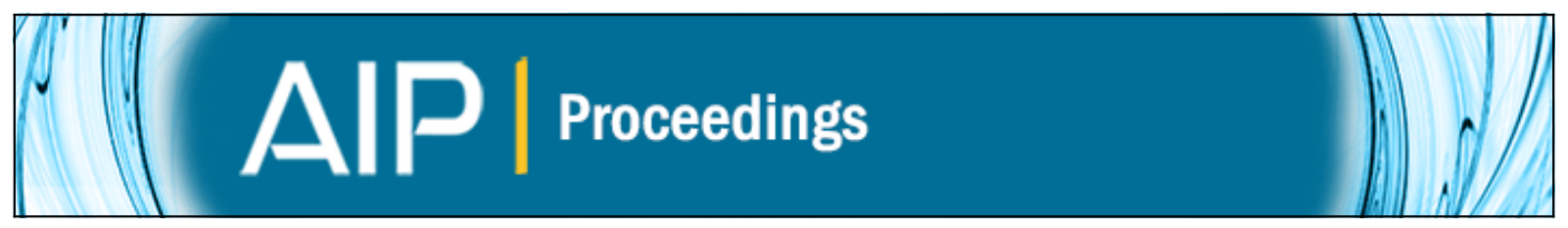

\title{
Energy conservation issues in the numerical solution of Hamiltonian PDEs
}

Luigi Brugnano, Gianluca Frasca Caccia, and Felice lavernaro

Citation: AIP Conference Proceedings 1648, 020002 (2015); doi: 10.1063/1.4912306

View online: http://dx.doi.org/10.1063/1.4912306

View Table of Contents: http://scitation.aip.org/content/aip/proceeding/aipcp/1648?ver=pdfcov

Published by the AIP Publishing

\section{Articles you may be interested in}

Recent advances in the numerical solution of Hamiltonian PDEs

AIP Conf. Proc. 1648, 150008 (2015); 10.1063/1.4912438

Energy conservation in the numerical solution of Hamiltonian boundary value problems

AIP Conf. Proc. 1558, 35 (2013); 10.1063/1.4825414

Convergence in numerical solutions of the Dicke Hamiltonian

AIP Conf. Proc. 1488, 418 (2012); 10.1063/1.4759424

A Cell-Based Finite Difference Method for the Numerical Solution of PDEs

AIP Conf. Proc. 1368, 69 (2011); 10.1063/1.3663462

Symposium on PDEs: Solutions and Asymptotics

AIP Conf. Proc. 1168, 1279 (2009); 10.1063/1.3241310 


\title{
Energy Conservation Issues in the Numerical Solution of Hamiltonian PDEs
}

\author{
Luigi Brugnano*, Gianluca Frasca Caccia* and Felice Iavernaro ${ }^{\dagger}$ \\ *Dipartimento di Matematica e Informatica "U. Dini", Università di Firenze, Italy \\ ${ }^{\dagger}$ Dipartimento di Matematica, Università di Bari, Italy
}

\begin{abstract}
In this paper we show that energy conserving methods, in particular those in the class of Hamiltonian Boundary Value Methods, can be conveniently used for the numerical solution of Hamiltonian Partial Differential Equations, after a suitable space semi-discretization.
\end{abstract}

Keywords: Hamiltonian Partial Differential Equations, Energy-conserving Runge-Kutta methods, Hamiltonian Boundary Value Methods. PACS: 02.60.-x; 45.20.dh; 45.20.Jj; 02.30.Hq; 02.70.Jn; 02.70.Bf. $\quad$ MSC: 65P10; 65L05; 65M20.

\section{INTRODUCTION}

Hamiltonian Partial Differential Equations (Hamiltonian PDEs) have been investigated since many years, especially in connection with the use of multi-symplectic methods $[1,13,14,15]$. In particular, under suitable boundary conditions, they are characterized by the conservation of the associated energy. After appropriate space discretization, one can still obtain a discrete Hamiltonian formulation of such equations, resulting into a corresponding discrete energy. This latter can be then conserved by using energy-conserving methods in the class of Hamiltonian Boundary Value Methods (HBVMs) [5, 6, 9], which are Runge-Kutta methods based on the concept of discrete line integral [10, 11, 12]. We shall sketch the approach when a finite difference space-discretization is considered and periodic boundary conditions are prescribed, even though the used approach can be generalized to the case of general boundary conditions [4]. As a prototypal problem, we consider the nonlinear wave equation, even though the arguments can be straightforwardly extended to other Hamiltonian PDEs,

$$
\begin{aligned}
u_{t t}(x, t) & =u_{x x}(x, t)-f^{\prime}(u(x, t)), \quad(x, t) \in(a, b) \times(0, \infty), \\
u(x, 0) & =\psi_{0}(x), \quad u_{t}(x, 0)=\psi_{1}(x), \quad x \in[a, b], \\
u(a, t) & =u(b, t), \quad t>0 .
\end{aligned}
$$

As usual, subscripts denote partial derivatives, and the functions $f, \psi_{0}$ and $\psi_{1}$ are supposed to be sufficiently smooth, so they define a regular solution $u(x, t)\left(f^{\prime}\right.$ denotes the derivative of $\left.f\right)$. By setting $v=u_{t}$, and defining the functional

$$
\mathscr{H}[u, v](t)=\int_{a}^{b}\left[\frac{1}{2} v^{2}(x, t)+\frac{1}{2} u_{x}^{2}(x, t)+f(u(x, t))\right] \mathrm{d} x \equiv \int_{a}^{b} E(x, t) \mathrm{d} x,
$$

we can rewrite (1) as the infinite-dimensional Hamiltonian system

$$
\begin{aligned}
& u_{t}(x, t)=v(x, t), \quad(x, t) \in(a, b) \times(0, \infty), \\
& v_{t}(x, t)=u_{x x}(x, t)-f^{\prime}(u(x, t)), \\
& u(x, 0)=\psi_{0}(x), \quad v(x, 0)=\psi_{1}(x), \quad x \in[a, b] \\
& u(a, t)=u(b, t), \quad t \geq 0 .
\end{aligned}
$$

i.e., the first-order formulation of (1), for which (2) is a conserved quantity, where the Hamiltonian formulation stems from the fact that $v=\frac{\delta \mathscr{H}}{\delta v}$ and $-u_{x x}(x, t)+f^{\prime}(u(x, t))=\frac{\delta \mathscr{H}}{\delta u}$, the functional derivatives of $\mathscr{H}$. 


\section{NUMERICAL APPROXIMATION}

For approximating problem (3), let us introduce the following discretization of the spatial variable,

$$
x_{i}=a+i \Delta x, \quad i=0, \ldots, N, \quad \Delta x=(b-a) / N,
$$

and the vectors:

$$
\mathbf{x}=\left(\begin{array}{c}
x_{0} \\
\vdots \\
x_{N-1}
\end{array}\right), \quad \mathbf{q}(t)=\left(\begin{array}{c}
u_{0}(t) \\
\vdots \\
u_{N-1}(t)
\end{array}\right), \quad \mathbf{p}(t)=\left(\begin{array}{c}
v_{0}(t) \\
\vdots \\
v_{N-1}(t)
\end{array}\right) \in \mathbb{R}^{N},
$$

with $u_{i}(t) \approx u\left(x_{i}, t\right), v_{i}(t) \approx v\left(x_{i}, t\right) \equiv u_{t}\left(x_{i}, t\right)$. Because of the periodic boundary conditions, we also set:

$$
u_{N}(t) \equiv u_{0}(t), \quad u_{-1}(t) \equiv u_{N-1}(t), \quad t \geq 0 .
$$

Approximating the second derivative in (3) as

$$
u_{x x}\left(x_{i}, t\right) \approx \frac{u_{i+1}(t)-2 u_{i}(t)+u_{i-1}(t)}{\Delta x^{2}}, \quad i=0, \ldots, N-1,
$$

yields the following semi-discrete problem,

$$
\dot{\mathbf{q}}=\mathbf{p}, \quad \dot{\mathbf{p}}=-\frac{1}{\Delta x^{2}} T_{N} \mathbf{q}-f^{\prime}(\mathbf{q}), \quad t>0,
$$

and the following approximation of the Hamiltonian (2),

$$
H(\mathbf{q}, \mathbf{p})=\Delta x\left[\frac{\mathbf{p}^{\top} \mathbf{p}}{2}+\frac{\mathbf{q}^{\top} T_{N} \mathbf{q}}{2 \Delta x^{2}}+\mathbf{e}^{\top} f(\mathbf{q})\right],
$$

where $\mathbf{e}=\left(\begin{array}{ccc}1 & \ldots & 1\end{array}\right)^{\top} \in \mathbb{R}^{N}$, and $T_{N}$ is, because of the periodic boundary conditions, a circulant matrix:

$$
T_{N}=\left[\begin{array}{ccccc}
2 & -1 & & & -1 \\
-1 & \ddots & \ddots & & \\
& \ddots & \ddots & \ddots & \\
& & \ddots & \ddots & -1 \\
-1 & & & -1 & 2
\end{array}\right] \in \mathbb{R}^{N \times N} .
$$

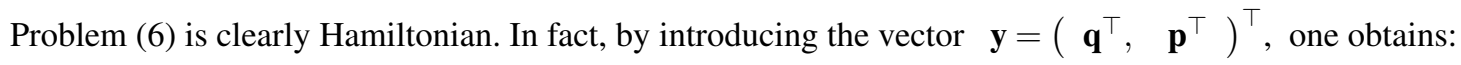

$$
\dot{\mathbf{y}}=J_{N} \nabla H(\mathbf{y}), \quad \text { with } \quad J_{N}=\frac{1}{\Delta x}\left(\begin{array}{cc}
I_{N} \\
-I_{N}
\end{array}\right),
$$

where here and in the sequel we use the notation $H(\mathbf{y})=H(\mathbf{q}, \mathbf{p})$. Consequently,

$$
\dot{H}(\mathbf{y})=\nabla H(\mathbf{y})^{\top} \dot{\mathbf{y}}=\nabla H(\mathbf{y})^{\top} J_{N} \nabla H(\mathbf{y})=0,
$$

because $J_{N}$ is skew-symmetric. One then concludes that the discrete approximation (7) is a conserved quantity for the semi-discrete problem (8). For solving such a Hamiltonian problem, one can consider the use of a $\operatorname{HBVM}(k, s)$ method, which is the $k$-stage Runge-Kutta method defined by the following Butcher tableau $[6,9]$ :

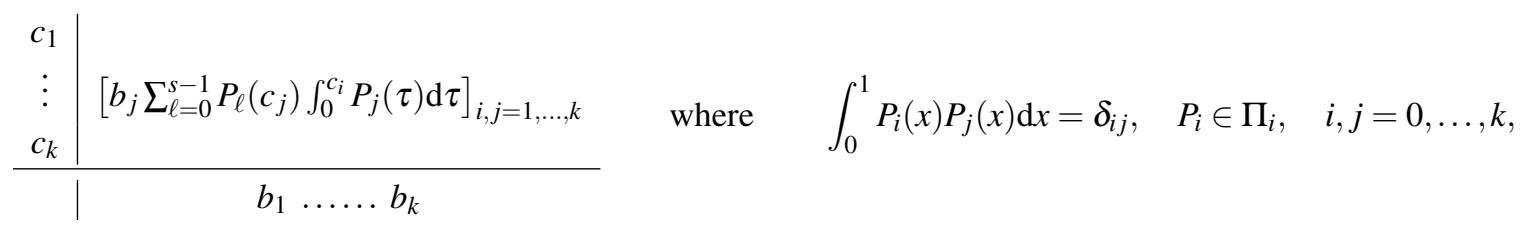


with $P_{i}, P_{j}$ the normalized and shifted Legendre polynomials, and $\left(b_{i}, c_{i}\right)_{i=1, \ldots, k}$ the Legendre weights and abscissae. For such methods, which reduce to the $s$-stage Gauss-Legendre collocation methods when $k=s$, the following result holds true [4].

Theorem 1 For all $k \geq s$, a HBVM(k,s) method applied to problem (7)-(8) with stepsize h:

- is symmetric and of order $2 s$;

- is energy conserving when $f$ is a polynomial of degree $v \leq\lfloor 2 k / s\rfloor$;

- for general and suitably regular functions $f$, the energy error at each step is $O\left(h^{2 k+1}\right)$.

This, in turn, implies that a (at least practical) conservation of energy is obtained, provided that $f$ is suitably regular (which will be assumed hereafter), by choosing $k$ large enough, also considering that the computational cost for implementing a $\operatorname{HBVM}(k, s)$ method depends essentially on $s$, rather than on $k[8,2,3]$.

We conclude this section, by stressing again that the above arguments can be extended to cope with different kind of boundary conditions, by suitably modifying the Hamiltonian function defining the problem [4].

\section{NUMERICAL TESTS}
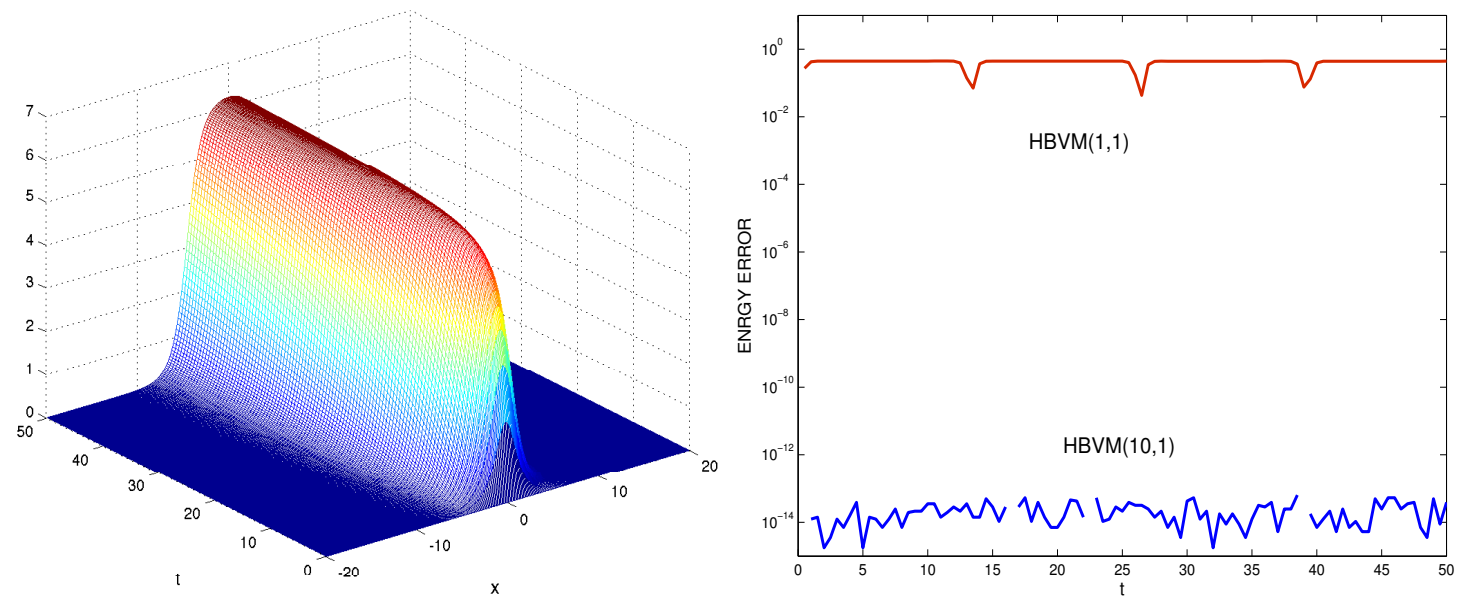

FIGURE 1.

Even though the use of energy-conserving methods is quite well understood, proving to be very useful, when speaking about Hamiltonian ordinary differential equations, their use in the framework of Hamiltonian partial differential equations is fairly less obvious and deserves further investigations. Nevertheless, we report here an example which should highlight the usefulness of using energy-conserving methods for solving Hamiltinian PDEs. In particular, let us consider the well-known sine-Gordon equation, with a soliton-like solution. Namely, (1) with

$$
-a=b=20, \quad f(u)=1-\cos u, \quad \psi_{0}(x) \equiv 0, \quad \psi_{1}(x)=4 \operatorname{sech} x,
$$

for which the Hamiltonian (2) has a value $H_{0} \approx 16$. The value of the Hamiltonian is practically matched by the discrete Hamiltonian (7), when using $N=400$ for the space discretization (4). In the left-plot in Figure 1, there is the correct solution $u(x, t)$ on the domain $[-20,20] \times[0,50]$. Such a shape changes suddenly for both larger and smaller values of the Hamiltonian: in other words, such a value is a separatrix for the underlying dynamics. Clearly, in the discrete setting the space discretization will cause the dynamics to eventually fall into either one of the two nearby regimes. Nevertheless, one reasonably expects that this will happen later, when using an energy-conserving method. For this purpose, in the right-plot in Figure 1 we plot the absolute error in the numerical Hamiltonian, when using the second-order $\operatorname{HBVM}(10,1)$ and the implicit mid-point (i.e., $\operatorname{HBVM}(1,1))$ methods, over the interval $[0,50]$, with stepsize $h=0.5$. As is clear, the energy error is negligible (i.e., of the order of the machine precision) for the former method, whereas it is not for the latter method. In the left-plot in Figure 2, is the numerical solution computed by 

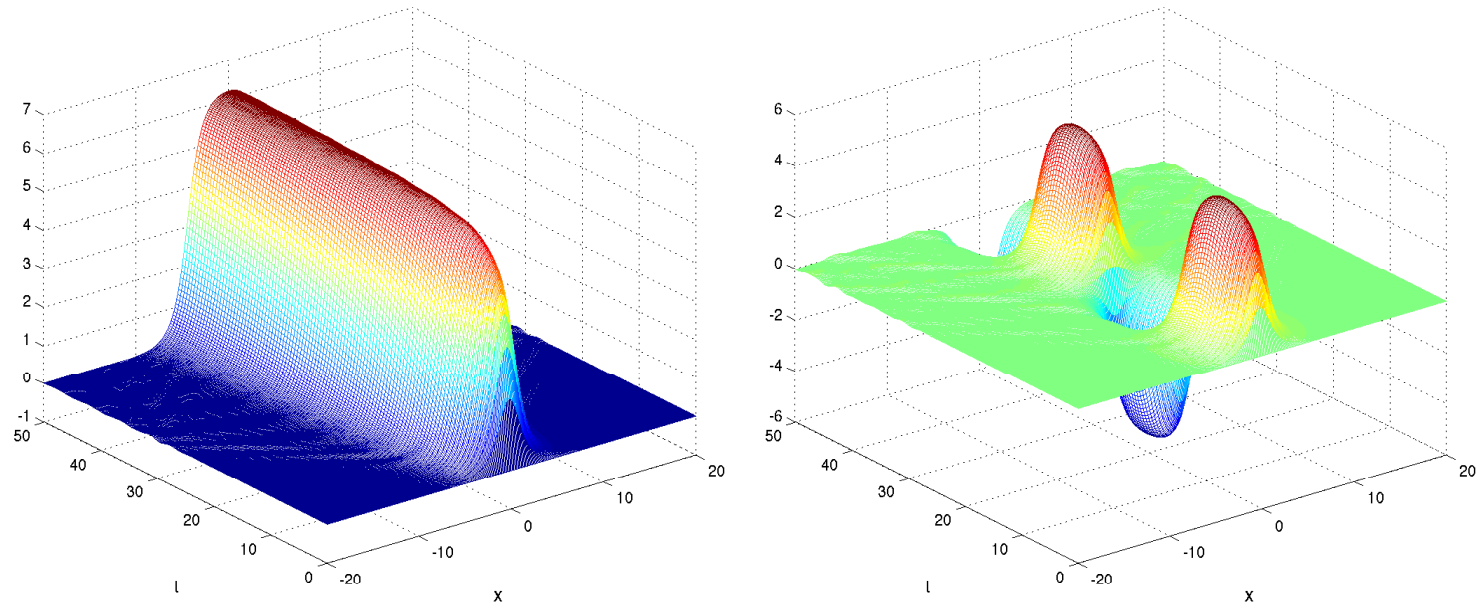

FIGURE 2.

$\operatorname{HBVM}(10,1)$, whereas in the right-plot, of the same figure, is the numerical solution computed by the (symplectic) implicit mid-point rule: as is clear, the former numerical solution has the correct shape, unless the latter one.

\section{REFERENCES}

1. T.J. Bridges, S. Reich. Numerical methods for Hamiltonian PDEs. J. Phys. A: Math. Gen. 39 (2006) 5287-5320.

2. L. Brugnano, G. Frasca Caccia, F. Iavernaro. Efficient implementation of geometric integrators for separable Hamiltonian problems. AIP Conf. Proc. 1588 (2013) 734-737.

3. L. Brugnano, G. Frasca Caccia, F. Iavernaro. Efficient implementation of Gauss collocation and Hamiltonian Boundary Value Methods. Numer. Algor. 65 (2014) 633-650.

4. L. Brugnano, G. Frasca Caccia, F. Iavernaro. Energy conservation issues in the numerical solution of the nonlinear wave equation. (submitted).

5. L. Brugnano, F. Iavernaro, D. Trigiante. Hamiltonian BVMs (HBVMs): a family of "drift-free" methods for integrating polynomial Hamiltonian systems. AIP Conf. Proc. 1168 (2009) 715-718.

6. L. Brugnano, F. Iavernaro, D. Trigiante. Hamiltonian Boundary Value Methods (Energy Preserving Discrete Line Integral Methods). J. Numer. Anal. Ind. Appl. Math. 5,1-2 (2010) 17-37.

7. L. Brugnano, F. Iavernaro, D. Trigiante. Analisys of Hamiltonian Boundary Value Methods (HBVMs): a class of energypreserving Runge-Kutta methods for the numerical solution of polynomial Hamiltonian systems. Communications in Nonlinear Science and Numerical Simulation http://dx.doi.org/10.1016/j.cnsns.2014.05.030

8. L. Brugnano, F. Iavernaro, D. Trigiante. A note on the efficient implementation of Hamiltonian BVMs. J. Comput. Appl. Math. 236 (2011) 375-383.

9. L. Brugnano, F. Iavernaro, D. Trigiante. A simple framework for the derivation and analysis of effective one-step methods for ODEs. Appl. Math. Comput. 218 (2012) 8475-8485.

10. F. Iavernaro, B. Pace. $s$-Stage trapezoidal methods for the conservation of Hamiltonian functions of polynomial type. AIP Conf. Proc. 936 (2007) 603-606.

11. F. Iavernaro, B. Pace. Conservative Block Boundary Value Methods for the Solution of Polynomial Hamiltonian Systems. AIP Conf. Proc. 1048 (2007) 888-891.

12. F. Iavernaro, D. Trigiante. High-order symmetric schemes for the energy conservation of polynomial Hamiltonian problems, J. Numer. Anal. Ind. Appl. Math. 4 (1-2) (2009) 87-101.

13. A.L. Islas, C.M. Schober. Backward error analysis for multisymplectic discretizations of Hamiltonian PDEs. Mathematic and Computers in Simulation 69 (2005) 290-303.

14. A.L. Islas, C.M. Schober. Conservation properties of multisymplectic integrators. Future Generation Computer Systems 22 (2006) 412-422.

15. B. Leimkulher, S. Reich. Simulating Hamiltonian Dynamics. Cambridge University Press, 2004. 